\title{
Memory loss in Alzheimer's disease: are the alterations in the UPR network involved in the cognitive impairment?
}

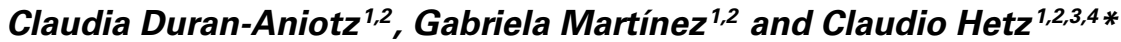 \\ ${ }^{1}$ Faculty of Medicine, Biomedical Neuroscience Institute, University of Chile, Santiago, Chile \\ 2 Program of Cellular and Molecular Biology, Center for Molecular Studies of the Cell, Institute of Biomedical Sciences, University of Chile, Santiago, Chile \\ ${ }^{3}$ Neurounion Biomedical Foundation, Santiago, Chile \\ ${ }^{4}$ Department of Immunology and Infectious diseases, Harvard School of Public Health, Boston, MA, USA \\ *Correspondence: chetz@hsph.harvard.edu or chetz@med.uchile.cl
}

Edited by:

Antonio Camins, University of Barcelona, Spain

Reviewed by:

Lei Mao, Charite, Germany

Maria Jose Silva Fernandes, Universidade Federal de São Paulo, Brazil

Keywords: Alzheimer's disease, UPR signaling pathways, memory, ER stress, memory impairment

Alzheimer's disease $(\mathrm{AD})$ is a progressive and devastating age-related neurodegenerative disorder, involving memory loss and the extracellular deposition in the brain of misfolded and aggregated amyloid beta $(A \beta)$ peptide (Holtzman et al., 2011). The molecular mechanism that triggers $\mathrm{AD}$ is not completely understood. The AD neuropathological process begins many years before the clinical onset with general alterations in protein homeostasis (referred to as proteostasis) among other effects. Recent evidence suggests that disturbances in the normal function of the secretory pathway and the occurrence of endoplasmic reticulum (ER) stress may represent a common pathological feature of familial and sporadic AD (Cornejo and Hetz, 2013). ER stress engages an adaptive reaction known as the unfolded protein response (UPR) which modulates many aspects of ER proteostasis to decrease the unfolded protein load (Walter and Ron, 2011). Under conditions of irreversible or chronic ER stress the UPR shifts its signaling toward induction of apoptosis.

$\mathrm{A} \beta$ oligomers are known to induce neuronal loss and dysfunction (Mucke and Selkoe, 2012) and impair synaptic plasticity and memory in animal models of $\mathrm{AD}$ (Cleary et al., 2005; Shankar et al., 2008). In this line, whether ER stress causes cognitive impairment remained poorly studied until very recently. Besides, interesting novel concepts are emerging where ER stress may actually operates upstream of the generation of $A \beta$ as part of the etiology of the disease (Yoon et al., 2012).
Could these findings provide insights about new points for disease intervention? Many recent studies have developed small molecules and gene therapy strategies to alleviate ER stress in vivo, which offers interesting future applications for the development of clinical trials in $\mathrm{AD}$ and other diseases (Hetz et al., 2013).

Medial temporal lobe areas, such as the hippocampus and entorhinal cortex, are the first regions affected during the progression of $\mathrm{AD}$, contributing to the occurrence of dementia in affected patients. Under diverse stress conditions, including ER stress, inhibition of protein synthesis operates as a survival pathway that is mediated by the phosphorylation of eukaryotic translation initiator factor $2 \alpha$ (eIF2 $\alpha$ ), referred to as the "integrated stress response." Of note, the process of memory consolidation and synaptic plasticity involve active protein synthesis, among other events (Costa-Mattioli et al., 2009). In fact, several studies have shown that exacerbated phosphorylation of eIF2 $\alpha$ induces cognitive impairment (Costa-Mattioli et al., 2005, 2009; Jiang et al., 2010). In agreement with this findings, an elegant recent study demonstrated that decreasing the expression of two of the eIF2 $\alpha$ kinases, double-stranded RNA-activated protein kinase (PKR)-like endoplasmic reticulum kinase (PERK) and General control non-derepressible-2 (GCN2), improve cognitive function and synaptic plasticity in an $\mathrm{AD}$ transgenic mouse model (Ma et al., 2013). In addition, targeting another eIF2 $\alpha$ kinase termed dsRNA-dependent protein kinase (PKR), can also improve learning and memory processes at basal levels (Zhu et al., 2011), similarly to GCN2 deficient animals. Consistent with these finding, another recent report demonstrated that brain inflammation in $\mathrm{AD}$ models engages PKR to induce synaptic loss and memory impairment (Lourenco et al., 2013). In that study the authors also showed that $A \beta$ oligomers alters insulin signaling leading to memory deficits through a mechanism involving the proinflammatory cytokine tumor necrosis factor (TNF)- $\alpha$. Of note, PERK deficiency in the nervous system did not alter learning and memory-related processes at basal levels, and only impacted cognition in the context of $\mathrm{AD}$ models when ER proteostasis is altered (Ma et al., 2013). Importantly, these results solved an important question since they indicated that despite of reducing the adaptive activity of one branch of the UPR on a model of $\mathrm{AD}$, this genetic manipulation improved cognitive aspects of $\mathrm{AD}$ without affecting the ability of cells to survive under the stress conditions generated by the accumulation of amyloid beta. Is the phosphorylation of eIF2 $\alpha$ a key converging event involved in neuropathology and cognitive impairment in $\mathrm{AD}$ ? Is this the molecular link between protein misfolding and neuroinflammation? These reports suggest the concept that modulation of protein synthesis through the eIF $2 \alpha$ axis is directly involved in memory formation and could be also exploited as a target to reduce synaptic dysfunction in $\mathrm{AD}$. 
Advances in this line were provided by a recent study identifying a small molecule called ISRIB that efficiently reduces the consequences of eIF2 $\alpha$ phosphorylation and improve learning and memory in wild-type rats (Sidrauski et al., 2013). This potent inhibitor showed promising pharmacokinetic properties, it crossed the blood-brain barrier with no overall adverse effects to the animal. These findings raise the possibility that compounds that inhibit PERK signaling may offer interesting future applications for the development of clinical trials in AD. Finetuning the concentrations the compounds will be a challenging issue due to the dual impact of this signaling pathway on cell fate. In this line, PERK inhibitors have been recently shown to revert synaptic dysfunction and neurodegeneration in models of Prion disease (Moreno et al., 2013).

The most conserved signaling pathways of the UPR network is initiated by the ER stress sensor IRE $1 \alpha$. Active IRE1 $\alpha$ splices the mRNA encoding the transcription factor X-box binding protein 1, shifting its coding reading frame that induces an active transcription factor termed XBP1s (Hetz, 2012). Last year, a polymorphism in the XBP1 promoter was described as a risk factor to develop AD. Remarkably, a global study to screen the universe of $\mathrm{XBP} 1$ s-target genes revealed that this factor regulates a cluster of $\mathrm{AD}$-related genes involved in the control of APP trafficking and processing (Acosta-Alvear et al., 2007). Together, these studies suggest that a second UPR signaling branch may also contribute to $\mathrm{AD}$ through a different mechanism. Although it was shown that cortical brain areas from post-mortem tissue showed a significant increase in the splicing of XBP1 mRNA (Lee et al., 2010), a recent report showed that XBP1 mRNA did not reach levels of healthy age-matched controls, suggesting down-regulation of this factor in AD brains (Reinhardt et al., 2013). In terms of functional studies, a neuro-protective activity of XBP1 was proposed on two fly models of $\mathrm{AD}$ involving the expression $\mathrm{A} \beta$ or Tau (Loewen and Feany, 2010; Casas-Tinto et al., 2011). Ectopic expression of XBP1s suppressed $\mathrm{A} \beta$ neurotoxicity in flies, possibly by modulating calcium homeostasis.

ER stress in $\mathrm{AD}$ also engages another stress pathways through IRE1 $\alpha$ governed

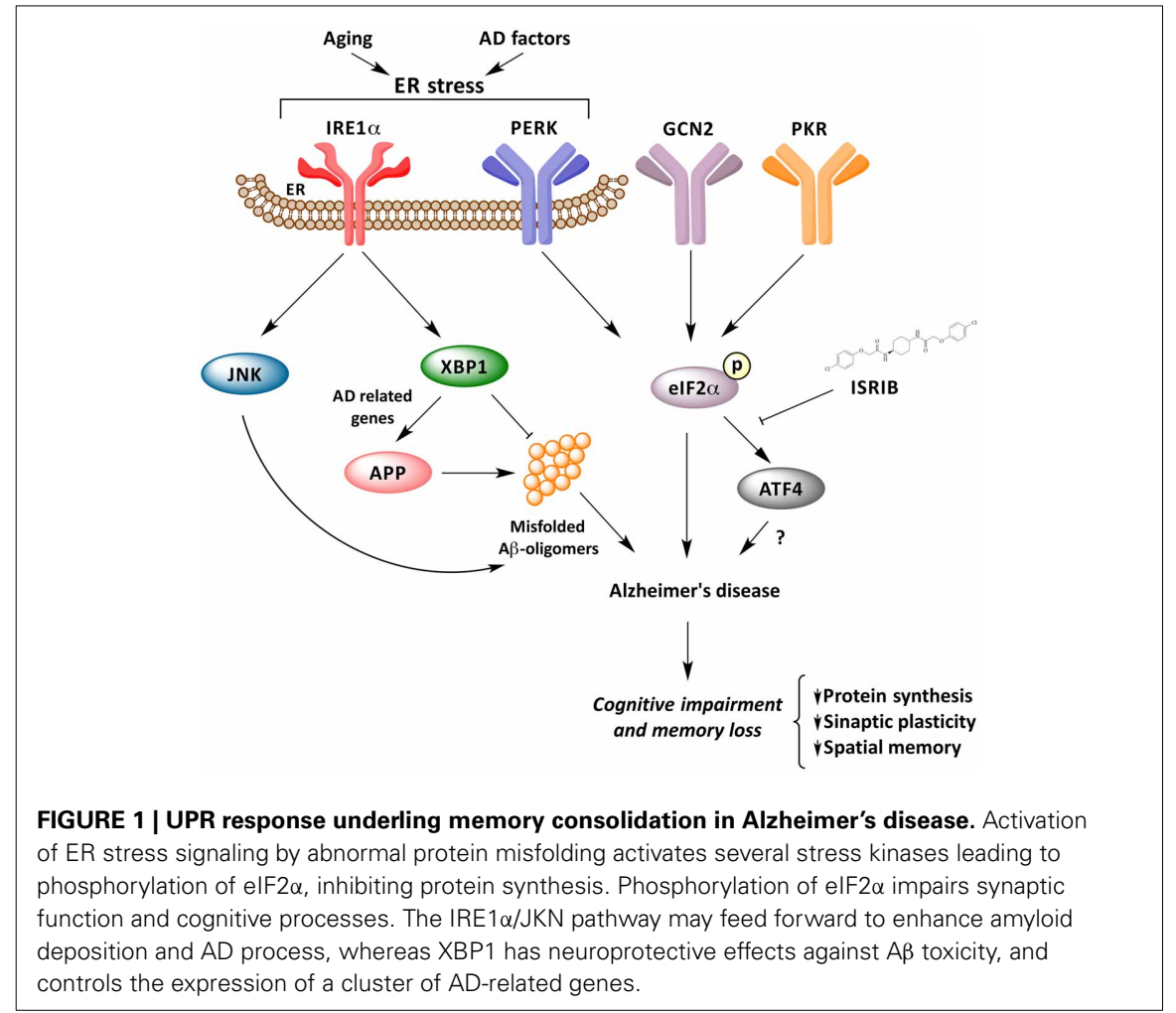

by cJun $\mathrm{N}$-terminal kinases (JNK). JNK is activated in neurons of $\mathrm{AD}$ post-mortem brain tissue, and a recent report proposed that the occurrence of ER stress in $\mathrm{AD}$ mouse models may positive feedback to enhance $A \beta$ formation and amyloid deposition through activation of JNK (Yoon et al., 2012). This study opens the question of whether ER stress signaling may contributes to diverse aspects of the disease: APP metabolism, A $\beta$ aggregation, neurodegeneration and cognitive impairment. These observations are interesting because they contrast with the results obtained after manipulation of the UPR in other disease models, where the pathway has protective effects against protein aggregation (Matus et al., 2011; Roussel et al., 2013). These findings highlight the need to systematically investigate the actual contribution of XBP1, IRE1, and other UPR components such as ATF6 to AD to further validate and define the exact contribution of this homeostatic pathway to the disease process. Still, the cause of abnormal ER stress in $\mathrm{AD}$ remains to be determined.

Many important questions are still open in this emerging and growing field: (i) Is the IRE $1 \alpha$ network, IRE $1 \alpha / \mathrm{XBP} 1$ and/or IRE $1 \alpha /$ JNK pathways, involved in the consolidation and formation of memory? (ii) Do the activation of these pathways play a functional role in cognitive decline in AD? and, (iii) How is the UPR network as a whole related to the progression and pathogenesis of $\mathrm{AD}, \mathrm{APP}$ processing and $A \beta$ oligomers generation? Is neuroinflammation also converging into the IREla UPR axis? How can we consolidate that PERK signaling may have a dual and opposing activity in AD? All of the available evidence points to the fact that ER disturbances and UPR activation may facilitate and amplify both memory loss and protein aggregation on a vicious cycle that may turn initial adaptive UPR responses into a pro-degenerative factor (Figure 1). A systematic analysis is required to assess the exact contribution of each UPR signaling branches to AD to then define optimal targets for disease intervention.

\section{ACKNOWLEDGMENTS}

This work was funded by the Alzheimer Disease Association, Millennium Institute no. P09-015-F, FONDECYT no. 1100176, ACT1109; CONICYT grant USA20130003, ECOS-CONICYTC13S02 and FONDEF D11I1007 (Claudio Hetz), 
Doctoral Fellow supported by CONICYT (Gabriela Martínez) and FONDECYT grant no. 3140466 (Claudia DuranAniotz).

\section{REFERENCES}

Acosta-Alvear, D., Zhou, Y., Blais, A., Tsikitis, M., Lents, N. H., Arias, C., et al. (2007). XBP1 controls diverse cell type- and condition-specific transcriptional regulatory networks. Mol. Cell 27, 53-66. doi: 10.1016/j.molcel.2007.06.011

Casas-Tinto, S., Zhang, Y., Sanchez-Garcia, J., Gomez-Velazquez, M., Rincon-Limas, D. E., and Fernandez-Funez, P. (2011). The ER stress factor XBP1s prevents amyloid-beta neurotoxicity. Hum. Mol. Genet. 20, 2144-2160. doi: 10.1093/hmg/ ddr 100

Cleary, J. P., Walsh, D. M., Hofmeister, J. J., Shankar, G. M., Kuskowski, M. A., Selkoe, D. J., et al (2005). Natural oligomers of the amyloid-beta protein specifically disrupt cognitive function. Nat. Neurosci. 8, 79-84. doi: 10.1038/nn1372

Cornejo, V. H., and Hetz, C. (2013). The unfolded protein response in Alzheimer's disease. Semin. Immunopathol. 35, 277-292. doi: 10.1007/s00281013-0373-9

Costa-Mattioli, M., Gobert, D., Harding, H., Herdy, B., Azzi, M., Bruno, M., et al. (2005). Translational control of hippocampal synaptic plasticity and memory by the eIF2alpha kinase GCN2. Nature 436, 1166-1173. doi: 10.1038/nature03897

Costa-Mattioli, M., Sossin, W. S., Klann, E., and Sonenberg, N. (2009). Translational control of long-lasting synaptic plasticity and memory. Neuron 61, 10-26. doi: 10.1016/j.neuron.2008. 10.055

Hetz, C. (2012). The unfolded protein response: controlling cell fate decisions under ER stress and beyond. Nat. Rev. Mol. Cell Biol. 13, 89-102. doi: $10.1038 / \mathrm{nrm} 3270$

Hetz, C., Chevet, E., and Harding, H. P. (2013). Targeting the unfolded protein response in disease. Nat. Rev. Drug Discov. 12, 703-719. doi: 10.1038/ $\operatorname{nrd} 3976$

Holtzman, D. M., Morris, J. C., and Goate, A. M. (2011). Alzheimer's disease: the challenge of the second century. Sci. Transl. Med. 3, 77sr71. doi: 10.1126/scitranslmed.3002369
Jiang, Z., Belforte, J. E., Lu, Y., Yabe, Y., Pickel, J., Smith, C. B., et al. (2010). eIF2alpha Phosphorylation-dependent translation in CA1 pyramidal cells impairs hippocampal memory consolidation without affecting general translation. J. Neurosci. 30, 2582-2594. doi: 10.1523/ JNEUROSCI.3971-09.2010

Lee, J. H., Won, S. M., Suh, J., Son, S. J., Moon, G. J., Park, U. J., et al. (2010). Induction of the unfolded protein response and cell death pathway in Alzheimer's disease, but not in aged Tg2576 mice. Exp. Mol. Med. 42, 386-394. doi: 10.3858/ emm.2010.42.5.040

Loewen, C. A., and Feany, M. B. (2010). The unfolded protein response protects from tau neurotoxicity in vivo. PLoS ONE 5:e13084. doi: 10.1371/journal.pone. 0013084

Lourenco, M. V., Clarke, J. R., Frozza, R. L., Bomfim, T. R., Forny-Germano, L., Batista, A. F., et al. (2013). TNF-alpha Mediates PKR-dependent memory impairment and brain IRS-1 inhibition induced by Alzheimer's beta-amyloid oligomers in mice and monkeys. Cell Metab. 18, 831-843. doi: 10.1016/j.cmet.2013.11.002

Ma, T., Trinh, M. A., Wexler, A. J., Bourbon, C., Gatti, E., Pierre, P., et al. (2013). Suppression of eIF2alpha kinases alleviates Alzheimer's diseaserelated plasticity and memory deficits. Nat. Neurosci. 16, 1299-1305. doi: 10.1038/nn.3486

Matus, S., Glimcher, L. H., and Hetz, C. (2011). Protein folding stress in neurodegenerative diseases: a glimpse into the ER. Curr. Opin. Cell Biol. 23, 239-252. doi: 10.1016/j.ceb.2011.01.003

Moreno, J. A., Halliday, M., Molloy, C., Radford, H., Verity, N., Axten, J. M., et al. (2013). Oral treatment targeting the unfolded protein response prevents neurodegeneration and clinical disease in prion-infected mice. Sci. Transl. Med. 5, 206 ral38. doi: 10.1126/scitranslmed.3006767

Mucke, L., and Selkoe, D. J. (2012). Neurotoxicity of amyloid beta-protein: synaptic and network dysfunction. Cold Spring Harb. Perspect. Med. 2:a006338. doi: 10.1101/cshperspect.a006338

Reinhardt, S., Schuck, F., Grosgen, S., Riemenschneider, M., Hartmann, T., Postina, R., et al. (2013). Unfolded protein response signaling by transcription factor XBP-1 regulates ADAM10 and is affected in Alzheimer's disease. FASEB J. 28, 1-20. doi: 10.1096/fj.13-234864
Roussel, B. D., Kruppa, A. J., Miranda, E., Crowther, D. C., Lomas, D. A., and Marciniak, S. J. (2013). Endoplasmic reticulum dysfunction in neurological disease. Lancet Neurol. 12, 105-118. doi: 10.1016/S1474-4422(12)70238-7

Shankar, G. M., Li, S., Mehta, T. H., Garcia-Munoz, A., Shepardson, N. E., Smith, I., et al. (2008) Amyloid-beta protein dimers isolated directly from Alzheimer's brains impair synaptic plasticity and memory. Nat. Med. 14, 837-842. doi: 10.1038/nm1782

Sidrauski, C., Acosta-Alvear, D., Khoutorsky, A., Vedantham, P., Hearn, B. R., Li, H., et al. (2013). Pharmacological brake-release of mRNA translation enhances cognitive memory. Elife 2:e00498. doi: 10.7554/eLife.00498

Walter, P., and Ron, D. (2011). The unfolded protein response: from stress pathway to homeostatic regulation. Science 334, 1081-1086. doi: 10.1126/science. 1209038

Yoon, S. O., Park, D. J., Ryu, J. C., Ozer, H. G., Tep, C., Shin, Y. J., et al. (2012). JNK3 perpetuates metabolic stress induced by abeta peptides. Neuron 75, 824-837. doi: 10.1016/j.neuron.2012.06.024

Zhu, P. J., Huang, W., Kalikulov, D., Yoo, J. W., Placzek, A. N., Stoica, L., et al. (2011). Suppression of PKR promotes network excitability and enhanced cognition by interferon-gamma-mediated disinhibition. Cell 147, 1384-1396. doi: 10.1016/j. cell.2011.11.029

Received: 16 December 2013; accepted: 12 January 2014; published online: 30 January 2014.

Citation: Duran-Aniotz C, Martínez G and Hetz C (2014) Memory loss in Alzheimer's disease: are the alterations in the UPR network involved in the cognitive impairment? Front. Aging Neurosci. 6:8. doi: 10.3389/ fnagi.2014.00008

This article was submitted to the journal Frontiers in Aging Neuroscience.

Copyright (C) 2014 Duran-Aniotz, Martínez and Hetz. This is an open-access article distributed under the terms of the Creative Commons Attribution License (CC BY). The use, distribution or reproduction in other forums is permitted, provided the original author(s) or licensor are credited and that the original publication in this journal is cited, in accordance with accepted academic practice. No use, distribution or reproduction is permitted which does not comply with these terms. 